\title{
A randomised crossover study to assess the usability of two new vision tests in patients with low vision.
}

Jasleen K. Jolly MSc MCOptom,2, Joanna M. Gray ${ }^{1}$ MBBS, A Paola Salvetti ${ }^{1,2}$, Ruofan C. Han BMBCh ${ }^{1,2}$, Robert E. MacLaren DPhil FRCOphth ${ }^{1,2^{*}}$

${ }^{1}$ Nuffield Laboratory of Ophthalmology, Nuffield Department of Clinical Neurosciences, University of Oxford, Oxford, United Kingdom; Oxford Biomedical Research Centre ${ }^{2}$ Oxford Eye Hospital, John Radcliffe Hospital, Oxford, United Kingdom

* Corresponding author. Nuffield Laboratory of Ophthalmology, Levels $5 \& 6$ John Radcliffe Hospital West Wing, Headley Way, Headington, Oxford OX3 9DU. Phone: 01865223380. Email address: enquiries@eye.ox.ac.uk

Financial: The research was funded by the National Institute for Health Research (NIHR) Oxford Biomedical Research Centre (BRC) based at Oxford University Hospitals NHS Trust and University of Oxford.

The views expressed are those of the authors and not necessarily those of the NHS, the NIHR or the Department of Health. The sponsor and funding organization had no role in the design or conduct of this research.

Word count 3080 words; 2 tables; 8 figures 


\section{Abstract}

Significance: Well established charts such as ETDRS are able to quantify visual acuity (VA) with a low cut-off of 1.6 logMAR. Below this point, non-quantitative measures, such as count fingers, hand movements and light perception are used. There is a need for more reproducible, comparable and reliable ways to measure VA changes in this patient cohort.

Purpose: To examine and compare the ability of the Berkeley Rudimentary Vision Test (BRVT) and the Freiburg Acuity Test (FrACT) to quantify VA in low vision patients who score non-numerical VAs in standard charts.

Methods: Fifty adult participants with VA $\leq 1.0 \log M A R$ in both eyes were recruited from the Oxford Eye Hospital, UK. Correlation between FrACT and BRVT results, as well as the correlation between VA and daily living activities were analysed statistically. Potential predictors of differences were investigated.

Results: BRVT was significantly faster to conduct $(P=0.002)$, but FrACT was able to quantify vision numerically in a greater proportion of eyes. The kappa agreement between tests was 0.26 . The difference increased systematically with the VA reduction $(P<0.0001)$. The Bland-Altman analysis showed a skew to measurement of lower logMAR VA indicating better vision measured on the FrACT. The only significant predictor of difference between the tests was binocular VA (coefficient $=-0.445, P=0.001$ ).

Conclusions: Both tests are suitable for a very low vision population. The BRVT is a faster test to administer, but FrACT provides a numerical result in more eyes. The poor inter-test repeatability indicates they cannot be used interchangeably. The BRVT generally reported poorer vision than the FrACT. The medium of presentation, such as a computer screen or externally lit print medium, is likely to be the biggest factor in these differences and warrants further investigation. 


\section{Introduction}

2 Visual acuity (VA) is defined as, "The capacity for seeing distinctly the details of an object" ${ }^{1}$ and

3 is the most established method of assessing visual function. VA represents the main endpoint

4 used in clinical trials as it is widely accepted by clinicians as well as by the regulatory

5 authorities. It is calculated as the reciprocal of the minimal angle of resolution in minutes of arc

6 at the eye. However, standardized letter charts like the well-established ETDRS often used in

7 clinical trials, have a low cut-off of $1.6 \log$ MAR. Below this point, non-quantitative measures of

8 VA, such as count fingers, hand movements and light perception are commonly used. This

9 established methodology has been shown to be highly variable, inaccurate and operator dependent in advanced disease stages. ${ }^{2,3}$ Additional charts have been developed to address

11 this gap in logMAR measurement. The Teller acuity cards were developed for use in infants and

12 children as well as low vision patients and had a lower limit of measurement of close to 2.0

13 logMAR depending on the working distance used. ${ }^{4}$ The original Bailey-Lovie logMAR charts

14 were designed to be used at $4 \mathrm{~m}$ but could measure down to 2.0 logMAR when used at closer 15 working distances. ${ }^{5}$

17 Recent novel interventions for sight restoration, such as electronic retinal implants and retinal gene therapy, have been targeted at very low vision populations unable to read the standard eye charts. Hence in addition to the requirement for more widespread provision of low vision rehabilitation services, ${ }^{6,7}$ we now need more reproducible and reliable ways to measure visual

21 acuity changes in this cohort of patients. ${ }^{8-10}$ The current measurements cannot detect effectively

22 the small but significant variation in vision that these patients may experience and often report

23 subjectively. To this end, there have been calls to set international guidelines for a more

24 accurate measure of vision when assessing the new technologies available. ${ }^{11}$ 
VA is considered one of the most critical assessments of vision as it is widely understood, as well as being used at every appointment for patient monitoring. Low vision interventions have been shown to improve patient quality of life ${ }^{6}$ However, with the inability to measure small but significant changes in vision in a very low vision population, the patients are not always offered additional help.

Although optotype based tests represent the backbone of VA assessments, there are several other types of VA measurements that might be more amenable to be used in a low vision population. The first reported test of acuity was star perception and the ability to pick out known constellations or celestial bodies. ${ }^{12}$ Grating assessments have been shown to produce equivalent results to optotypes and can be measured in logMAR scoring. ${ }^{13}$ This is due to the

fact they both determine the minimal angle of resolution, though optotypes add in a component of recognition. Optotypes requiring gap recognition, rather than identification, are a valid alternative..$^{14,15} \mathrm{An}$ attempt was made at measuring low vision by formalizing assessment of different aspects of light and motion perception. However, these tests did not produce a measure of vision in logMAR that was comparable to other visual acuity tests. ${ }^{16}$ Finally, grading of the identification of objects of known size might be a practical solution for very low visual acuity. ${ }^{17}$ However, once again these measurements do not provide a visual acuity grading equivalent to those currently in use. Several threshold analysis methods have been also tested, but are not directly interchangeable. ${ }^{18,19}$

\section{The Berkeley Rudimentary Vision Test (BRVT, Precision Vision, Woodstock, IL, USA) and} Freiburg Acuity Test (FrACT, Gundelfingen, Germany) are two different visual acuity systems developed to address the requirements of the very low vision population..$^{20-22}$ They have both been validated against the gold standard ETDRS charts. The measurable range of visual performance tested has also been extended to a logMAR value of 3.0 for FrACT and 2.9 for the 
52 BRVT. ${ }^{22,23}$ Both the BRVT and FrACT utilize a staircase probit threshold methodology and are

53 gaining popularity. The ability of tests to quantify vision in the very low vision population, and

54 their interchangeability was therefore investigated in more detail in the current study.

55 


\section{Methods}

57 This work conformed with the tenets of the Declaration of Helsinki, and prior informed consent was obtained from the participants. The study was approved by the NHS Research Ethics Committee (13/SC/0234). Fifty participants were recruited from Ophthalmology outpatient clinics in Oxford University Hospitals NHS Foundation Trust, Oxford, UK. Inclusion criteria were: Snellen visual acuity (VA) (or equivalent) $\leq 6 / 60$ in both eyes; age 18 or over; capacity to give informed consent and to undertake vision tests. Exclusion criteria were: vision loss without a confirmed anatomical cause; unwilling or unable to sign the informed consent and to complete the vision tests; VA better than 1.0 logMAR in either eye.

A questionnaire, developed with the aid of a focus group from the Oxfordshire Association for the Blind, covered fine and gross daily living activities at two light levels. The tasks were categorized into four broad categories of 'navigation', 'household', 'administrative', and 'social'. Participants were asked to answer 44 questions with 'yes', 'no' or 'not applicable' (Appendix 1). Each task answered yes was given a score of 1 . This was converted to a percentage of tasks scored yes.

Questionnaires were completed with the investigator's support to read out the questions. This allowed clarification of the meaning of the questions and the ascertain the participants' ability to perform tasks using only the visual function (i.e. not with the aid of touch, sound or using other strategies). After the questionnaire, patients underwent vision testing by both the Freiburg Acuity Test (FrACT) and Berkeley Rudimentary Vision Test (BRVT) tests on right eye first, followed by left eye and both eyes (FrACT only). The order of the monocular vision tests was randomized by a predetermined order which was unlocked only at the point of enrolment to remove possible confounding impact of a learning effect. 
82 FrACT testing was conducted using the $\mathrm{E}$ target in order to closely resemble the BRVT test as

83 much as possible. ${ }^{24}$ The test was run from a laptop attached to a 32 inch LCD screen with a

84 resolution of $1920 \times 1200$. White tape was added around the edge of the screen so the black

85 edge did not provide extra contrast that is not available in the BRVT. The initial testing distance

86 was $100 \mathrm{~cm}$ and the software was calibrated accordingly. Maximum acuity was set to unlimited,

$87100 \%$ optotype contrast, no crowding inducers and full screen presentation. Room illumination

88 was set to 3 lux with screen luminance set between 80 and $320 \mathrm{~cd} / \mathrm{m}^{2}$, as per the manufacturer

89 guidance. Each optotype was displayed for up to 60 seconds and participant response was according to a 4 forced choice paradigm. If the targets could not be identified, the screen was moved to $50 \mathrm{~cm}$ and the software recalibrated. If the optotypes could not be identified at $50 \mathrm{~cm}$ the viewing distance was adjusted to $25 \mathrm{~cm}$ and the software recalibrated. This test procedure allowed a maximum measurement of logMAR 3.0 with the screen and settings used.

The BRVT cards were presented in normal room illumination against a white background, initially at $1 \mathrm{~m}$. The mean luminance at the level of the participant was $240 \mathrm{~cd} / \mathrm{m}^{2}$. A flowchart for

97 the administration of the BRVT testing was designed based on manufacturer's guidelines and forms the basis of the vision measurement (Appendix 2). The BRVT had a maximum numerical reading of logMAR 2.90. The measured contrast of the 2 tests is provided in Appendix 3.

Statistical tests were carried out in $\mathrm{R}$ studio (version 10.4.3., supported by $\mathrm{R}$ 2.1.1). BlandAltman charts were constructed in SigmaPlot 13 (Systat Software Inc, CA, USA). Normality testing was performed using the functions (shapiro.test) and (qqplot), Mann-Witney $U$ using the function (wilcox.test), t-tests using the function (t.test), and multiple linear regression using the 105 linear regression function $(\mathrm{Im})$ in $\mathrm{R}$ studio version (Version 1.0.44, supported by R version 106 3.2.2). Correlation between the FrACT and BRVT tests in terms of time taken for tests, VA, and 107 possible confounders (age) was examined. 


\section{Results}

109 One hundred eyes from 50 patients were assessed. The age range was between 31 and 95 110 with a median age of 63.5 years. The cohort consisted of 30 female and 20 male patients (Table 111 1). The causes of vision loss are shown in Figure 1. On the basis of the diagnoses, we divided 112 the participants into two subgroups; primary peripheral or primary central vision loss. In total 24 113 participants were classified as 'peripheral' (those with retinal dystrophies and glaucoma), and 19 114 as 'central' (those with diabetic macular edema, Stargardt disease and age-related macular 115 degeneration). The remaining 7 patients had endophthalmitis, vascular problems, keratoconus 116 or retinal detachment. Nurse-recorded visual acuities (VA) ranged from 1.0 to perception of 117 light.

The raw data for right eyes in Figure $2 \mathrm{~A}$ and left eyes in Figure 2B, with non-measurable eyes being assigned a logMAR value of 3.5 . This value was chosen as it is outside the measurable range of both tests. The axes scale reflect the measurement range available on both tests. A

122 floor effect can be seen for the BRVT. On the BRVT, the measurement at VAs of 2.3logMAR or 123 higher may be from the grating acuity cards rather than tumbling $E$ targets. This introduces 124 variation from FrACT conditions and have been marked with separate markers to the main 125 dataset.

127 Of the 100 eyes tested, 70 had non-numerical VAs recorded in the notes. As shown in Table 2, 12823 were counting fingers, 27 hand movement and 20 had light perception. Both the Freiburg 129 Acuity Test (FrACT) and the Berkeley Rudimentary Vision Test (BRVT) gave numerical VAs for 130 all the 23 count finger eyes, but only for 20 hand movement eyes and 2 light perception eyes. In 1316 out of the hand movement eyes and 18 of the light perception eyes, the FrACT alone gave 132 numerical visual acuities. One hand movement eye did not score numerically on either the 133 FrACT or BRVT. The kappa agreement between FrACT and BRVT tests for ungradeable eyes, 
as tested by Fisher's Exact $r$ and c contingency table analysis test was 0.26 (low to fair) with a significant trend across qualitative measurement categories as defined by count fingers, hand movements and light perception $(P<0.0001)$. The difference between the tests increased systematically with the reduction in VA.

Test times were summed for the right and left eyes in order to analyze time taken for each test per patient. BRVT was faster to administer than FrACT (Wilcoxon signed rank test, $P=0.002$ ), taking a median time of 7.0 minutes (range 1.0 to 18.0 minutes) compared to 9.0 minutes for the FrACT (range 2.8 - 30.0 minutes) (Figure 3).

All the eyes in which the numerical VA could not be recorded in both tests were removed from further analysis, leaving 75 eyes in total. Two-thirds of the unquantifiable eyes had retinitis pigmentosa, indicating that poor peripheral vision may have a greater impact on the ability to perform the BRVT compared to the FrACT. Bland-Altman analysis was used to calculate VA agreement between tests for each eye separately (Figure 4A and B). The limits of agreement, as defined by $1.96 x$ standard deviation, were 0.63 and $0.68 \log$ MAR for the right and left eyes respectively. The Bland-Altman shows a skew in the mean difference between the tests of -0.22 and $-0.13 \log M A R$ in the right and left eyes respectively, indicating lower logMAR measurement (better vision) measured on the FrACT compared to the BRVT. As the FrACT records to the nearest 0.02 and the BRVT to the nearest 0.2 , we then re-analyzed the data, reclassifying the FrACT visual acuities to the nearest 0.2 using mathematical rounding in order to match the BRVT limits of 0.2 measurement interval. The limits of agreement were 0.44 to $-0.89 \log M A R$ in the right eye and 0.66 to $-0.98 \log M A R$ in the left eye. The limits of agreement remain similar to those found above indicating that the classification interval was not a cause for the difference. 
In order to understand the nature of the difference between FrACT and BRVT scores, a

160

stepwise multiple linear regression model was created using the right eye data, with age, binocular VA, gender, and type of visual loss (central, peripheral or other), as covariates. The only factor that was kept in the model was binocular VA (coefficient $=-0.445, P=0.001$ ) due to lack of independence between the other factors. The BRVT tended to show higher VA (poorer vision) than the FrACT in eyes with central vision loss compared to eyes with primary peripheral vision loss, mean VA difference 0.32 versus 0.04 logMAR.

The relationship between monocular and binocular VA in the FrACT test was examined. The Bland Altman plot showed that a difference of 0.40 logMAR between monocular and binocular conditions has a $95 \%$ chance of being within test-retest variability (Figure $5 \mathrm{~A}$ ). Once the two outlying points were removed, the limits of agreement reduced to 0.19 . The spread of VA measured using the FrACT was plotted separately for patients with central and peripheral vision loss (Figure $5 \mathrm{~B}$ ) and did not show any difference in spread in the visions measured in either eye.

Finally, we examined the relationship between binocular VA, as measured by FrACT score, and questionnaire performance. The questionnaire comprised of 44 questions, covering 4 broad domains of daily living activities: navigation (i.e. walking around in a park), household (i.e. cooking, choosing clothes), administrative tasks (i.e. reading and writing), and social tasks (i.e. recognizing faces, going to the theater.) Each domain comprised of a mix of 20 gross and 23 fine visual tasks. One question was unclassifiable as it pertained to 'hobbies', which varied in nature between participants (Figure 6A). Participants answered all 44 questions for bright (room) and dim light conditions. 
184 Surprisingly, we found that visual acuity and questionnaire performance were inversely

185 correlated: the worse the visual acuity (i.e. the higher the FrACT score with binocular testing or

186 BRVT score in the better eye), the better the performance on the questionnaire (Figure 6B).

187 Two-way ANOVA testing showed a significant difference when VA measured higher than 2.0

188 compared to lower than $2.0(P<0.0001)$ but there was no difference between FrACT and BRVT

$189(P=0.31)$. A waterfall chart was plotted to assess the individual responses to the

190 questionnaires. Each bar represents an individual's positive responses (expressed as a

191 percentage) to questionnaire tasks relating to navigation (red boxes), household (green boxes),

192 administrative (yellow boxes), and social tasks (blue boxes). The aim of the waterfall chart is to

193 see if there is any clustering of positive responses: for example, if we see a preponderance of

194 red bars to the left of the chart, this would suggest individuals generally are better at fulfilling

195 navigation-related tasks than tasks in other domains. In this case, there is a generally even

196 distribution of colors and thus task domains throughout the waterfall plot, with some clustering of

197 blue bars to the left of the chart. This indicates that individuals' ability to perform social tasks,

198 such as facial recognition, is least affected by poorer vision (Figure 7). ${ }^{25}$ 


\section{Discussion}

202 Standardizing the measurement of very low vision has become critically important in assessing

203 the novel therapeutic interventions that are becoming available in this patient group. Clinical trial

204 results are often reported in terms of letter scoring as used by ETDRS charts. ${ }^{26}$ However, this is

205 not a feasible strategy in the very low vision population, where the range of vision tested is

206 outside the limits of the ETDRS charts. Alternative strategies must therefore be explored. Both

207 the Freiburg Acuity Test (FrACT) and the Berkeley Rudimentary Vision Test (BRVT) are able to

208 extend the logMAR range of measurable vision and to establish the minimal angle of resolution

209 by using optotypes and gratings. Although the scoring system is altered, this extension provides

210 a valuable and important addition to the visual acuity (VA) scale, allowing differences in vision

211 that impact on patient quality of life to be detected.

212

213 To this end, two well established tests, the FrACT and BRVT, were compared in a clinical

214 population. They are based on the similar stimuli and principles, but differ in the presentation

215 platform. The FrACT is a computer- and the BRVT is a card-based test. The FrACT is presented

216 on a computer screen in a darkened room, whereas standard room lighting is used to illuminate

217 the BRVT cards externally. The difference between paper and screen formats has gained

218 interest for reading tasks, particularly in the field of education research. ${ }^{27}$ Although

219 comprehension appears to be slower in the screen format, higher luminance is required for the

220 paper readers to achieve equivalent vision to traditional screens. ${ }^{28}$ Visual acuity and reading

221 speed are, in fact, higher in internally lit scenarios, such as with a computer screen. In addition,

222 there is a reduction in the measurement variability in low vision patients with internal lighting,

223 indicating that the type of illumination is as important as the amount of illumination in visual

224 processing. ${ }^{29}$ The computer screen appears to present a more uniform contrast that holds at

225 different distances. This may explain why the tests were not found to be interchangeable and

226 we would encourage careful evaluation of lighting conditions to be used with the BRVT in future 
studies. Further standardization of the BRVT lighting may allow the tests to become equivalent, but may reduce the usability in a clinical setting.

Bland-Altman analysis shows a poor correlation between the tests. The difference between the tests was much larger than 0.2 logMAR, the maximum clinically acceptable difference used in clinical trials to denote what is considered test-retest change and what is considered a real change. ${ }^{30}$ This standard has been adopted by the Food and Drug Administration (FDA) to assess all ophthalmic and optical interventional trials. ${ }^{31}$ The FrACT usually returned a higher level of VA (better vision). Potential reasons for this discrepancy, such as measurement interval difference was explored, but did not appear to play a significant role. The BRVT uses a combination of the E optotype and gratings, whereas the FrACT uses exclusively E optotypes. Based on the spread of the data in Figure 1 this does not appear to account for the difference between the tests but could not be formally statistically tested due to the small number of datapoints in this range. Gratings have previously been reported to be equivalent to letter optotypes in the VA measures obtained. ${ }^{13}$ The internal lighting from the computer screen on the FrACT and increase in contrast may account for the difference between the two tests but difference in luminance of the 2 tests was not quantified. Further standardization of the BRVT lighting at a higher level may allow the tests to become equivalent, but would reduce the usability in a clinical setting.

In theory, both tests are capable of measuring VA up to a maximum of logMAR 2.90 / 3.0 on the set up used in this study. However, the FrACT measures higher numerical values (better vision) of logMAR VA, whereas the BRVT measures lower numerical values (worse vision) of logMAR VA. The FrACT was able to quantify more eyes. In addition, the FrACT provides a more precise measurement. It is also more flexible as it can be conducted on any screen or through a projector for larger stimuli. However, it requires calibration and a more involved setup, so the 
BRVT lends itself to a busy clinical setting or situations where portability is an asset. The BRVT was very well tolerated by patients, possibility due to the familiarity with a chart format. This may change in the next generation of patients who are accustomed to screens from a young age.

We found no consistent relationship between binocular and monocular VA. This is in line with previous reports. ${ }^{32}$ This may also be reflective of the difficulties in reliably measuring low vision. ${ }^{3}$ Self-reported visual capabilities have previously shown to be related to clinical measurements, but with a lot of variability. Interestingly, according to the questionnaire, poorer binocular VA appeared to be correlated with better daily living function but this was a weak correlation. Szlyk et al found the opposite, with a correlation between worse vision and a worse perception of abilities as would be expected intuitively. ${ }^{33}$ The change in this pattern may indicate that behavioural and psychological adaptation plays a major role in performance perception. It may be that the patients with worse visual acuities (who tended to have progressive diagnoses from a younger age, such as retinitis pigmentosa) have made behavioural adaptations and are

267 picking up on non-visual cues to help them with visually directed tasks. The variability observed 268 in both this study and Szlyk et al may be a reflection of a different adaptation to visual loss to 269 the point where patients are unable to differentiate if the tasks are performed by vision or by other mechanisms. Additionally, there is a question mark over how fixed is the traditional model

271 of cortical plasticity changes in the presence of vision loss. Adaptation may involve changes in

272 the neural processing of the remaining vision and help to explain some of the variability. ${ }^{34}$

273 Magnetic resonance imaging is being explored to investigate brain changes in the presence of 274 eye disease giving rise to the possibility that patients are unable to differentiate between visual 275 and non-visual cues once adapted to the vision loss. ${ }^{35}$

277 The inconclusive questionnaire results show the importance of using a standardized instrument 278 for measuring daily living skills. Rasch analysis on the questionnaire may improve the reliability 
279 of the results obtained by ensuring the questions are better targeted for the intended effect. ${ }^{36}$

280 The design of the questionnaire is likely a factor in the surprising results. However, since the

281 inception of this study, multiple validated quality of life instruments have been developed for the

282 low vision population and should ideally be used for all future work.

283 In summary, the FrACT and BRVT tests are both suitable for VA measurements in a low vision

284 population. The figures obtained from each test are not interchangeable and further work on test

285 lighting standardization is required to make them comparable.

286

287

288 Acknowledgements

289 We would like to thank Tallulah Shepherd for her contribution to data collection.

290

291 Appendix 1

292 Copy of the questionnaire used during the study.

293

294 Appendix 2

295 Flow chart showing the vision testing protocol for the Berkeley Rudimentary Vision Test (BRVT) 296 and providing a scoring guide.

297

\section{Appendix 3}

299 Contrast measurements of the tests in situ at different test distances. The contrast was

300 calculated by measuring dark and light areas on the charts under the illumination used for

301 testing as specified in the methods. This was done using a light meter, Seikonic L-758Cine

302 DigitalMaster (Seikonic Corporation Japan) then calculating the Weber contrast. A large $E$ was

303 used in both tests for the measurement. 


\section{References}

1. Millodot M. Dictionary of Optometry and Visual Science. 5th ed. Oxford: ButterworthHeinemann; 2000.

2. Lim L, Frazer D, Jackson A. Clinical Studies: Visual Acuities Beyond Snellen. Br J Ophthalmol 2007;92:153.

3. Kiser AK, Mladenovich D, Eshraghi F, et al. Reliability and Consistency of Visual Acuity and Contrast Sensitivity Measures in Advanced Eye Disease. Optom Vis Sci 2005;82:946-54.

4. Mash C, Dobson V. Long-Term Reliability and Predictive Validity of the Teller Acuity Card Procedure. Vision Res 1998;38:619-26.

5. Bailey IL, Lovie-Kitchin JE. Visual Acuity Testing. From the Laboratory to the Clinic. Vision Res 2013;90:2-9.

6. Lamoureux EL, Pallant JF, Pesudovs K, et al. The Effectiveness of Low-Vision Rehabilitation on Participation in Daily Living and Quality of Life. Invest Ophthalmol Vis Sci 2007;48:1476-82.

7. Edwards TL, Cottriall CL, Xue K, et al. Assessment of the Electronic Retinal Implant Alpha AMS in Restoring Vision to Blind Patients with End-Stage Retinitis Pigmentosa. Ophthalmology 2017:1-12.

8. Stingl K, Bartz-Schmidt KU, Besch D, et al. Subretinal Visual Implant Alpha IMS Clinical Trial Interim Report. Vision Res 2015;111:149-60.

9. Dorn JD, Ahuja AK, Caspi A, et al. The Detection of Motion by Blind Subjects With the Epiretinal 60-Electrode (Argus II) Retinal Prosthesis. JAMA Ophthalmol 2013;131:183-9.

10. Brandli A, Luu CD, Guymer RH, Ayton LN. Progress in the Clinical Development and Utilization of Vision Prostheses: An Update. Eye Brain 2016;8:15-25.

11. Rizzo JF, Ayton LN. Psychophysical Testing of Visual Prosthetic Devices: A Call to Establish a Multi-National Joint Task Force. J Neural Eng 2014;11:1-3. 
12. Bohigian GM. An Ancient Eye Test-Using the Stars. Surv Ophthalmol 2008;53:536-9.

13. Bittner AK, Jeter P, Dagnelie G. Grating Acuity and Contrast Tests for Clinical Trials of Severe Vision Loss. Optom Vis Sci 2011;88:1153-63.

14. Heinrich SP, Bach M. Resolution Acuity Versus Recognition Acuity with Landolt-Style Optotypes. Graefe's Arch Clin Exp Ophthalmol 2013;251:2235-41.

15. Miwa M, Iwanami M, Oba MS, et al. Comparison of LogMAR Eye Charts with Angular Vision for Visually Impaired: The Berkeley Rudimentary Vision Test vs LogMAR One Target Landolt Ring Eye Chart. Graefe's Arch Clin Exp Ophthalmol. 2013;251:2761-7.

16. Bach M, Wilke M, Wilhelm B, et al. Basic Quantitative Assessment of Visual Performance in Patients with Very Low Vision. Invest Opthalmol Vis Sci 2010;51:125560.

17. Finger RP, McSweeney SC, Deverell L, et al. Developing an Instrumental Activities of Daily Living Tool as Part of the Low Vision Assessment of Daily Activities Protocol. Invest Ophthalmol Vis Sci 2014;55:8458-66.

18. Sakuma JT, da Silva JA, Velasco e Cruz AA. Measurement of Visual Acuity with Logarithmic Charts: Comparison Between Methods of Threshold Estimation. Optom Vis Sci 1991;68:666-9.

19. Vanden Bosch ME, Wall M. Visual Acuity Scored by the Letter-By-Letter or Probit Methods has Lower Retest Variability Than the Line Assignment Method. Eye. $1997 ; 11: 411-17$

20. Bach M. The Freiburg Visual Acuity Test--Automatic Measurement of Visual Acuity. Optom Vis Sci 1996;73:49-53.

21. Schulze-Bonsel K, Feltgen N, Burau H, et al. Visual Acuities "Hand Motion" and "Counting Fingers" can be Quantified with the Freiburg Visual Acuity Test. Invest Ophthalmol Vis Sci 2006;47:1236-40. 
22. Bailey IL, Jackson a. J, Minto H, et al. The Berkeley Rudimentary Vision Test. Optom Vis Sci 2012;89:1257-64.

23. Knudsen LL. Visual Acuity Testing in Diabetic Subjects: The Decimal Progression Chart Versus the Freiburg Visual Acuity Test. Graefe's Arch Clin Exp Ophthalmol 2003;241:615-8.

24. Bach M. The Freiburg Visual Acuity Test-Variability Unchanged by Post-hoc Reanalysis. Graefe's Arch Clin Exp Ophthalmol. 2007;245:965-71.

25. Gillespie TW. Understanding Waterfall Plots. J Adv Pr Oncol 2012;3:106-11.

26. Edwards TL, Jolly JK, Groppe M, et al. Visual Acuity after Retinal Gene Therapy for Choroideremia. N Engl J Med. 2016;374:1996-8.

27. Mangen A, Walgermo BR, Brønnick K. Reading Linear Texts on Paper Versus Computer Screen: Effects on Reading Comprehension. Int J Educ Res 2013;58:61-8.

28. Lee DS, Ko YH, Shen IH, Chao CY. Effect of Light Source, Ambient Illumination, Character Size and Interline Spacing on Visual Performance and Visual Fatigue with Electronic Paper Displays. Display 2011;32:1-7.

29. Feng HL, Roth DB, Fine HF, et al. The Impact of Electronic Reading Devices on Reading Speed and Comfort in Patients with Decreased Vision. J Ophthalmol $2017 ; 3584706$.

30. Rosser DA, Cousens SN, Murdoch IE, et al. How Sensitive to Clinical Change are ETDRS LogMAR Visual Acuity Measurements? Invest Ophthalmol Vis Sci 2003;44:3278-81.

31. Kaiser PK. Prospective Evaluation of Visual Acuity Assessment: A Comparison of Snellen Versus ETDRS Charts in Clinical Practice (An AOS Thesis). Trans Am Ophthalmol Soc 2009;107:311-24. 
32. Rubin GS, Munoz B, Bandeen-Roche K, West SK. Monocular Versus Binocular Visual Acuity as Measures of Vision Impairment and Predictors of Visual Disability. Invest Ophthalmol Vis Sci 2000;41:3327-34.

33. Szlyk JP, Seiple W, Fishman GA, et al. Perceived and Actual Performance of Daily Tasks: Relationship to Visual Function Tests in Individuals with Retinitis Pigmentosa. Ophthalmology 2001;108:65-75.

34. Cheung S-H, Legge GE. Functional and Cortical Adaptations to Central Vision Loss. Vis Neurosci 2005;22:187-201.

35. Brown HDH, Woodall RL, Kitching RE, et al. Using Magnetic Resonance Imaging to Assess Visual Deficits: A Review. Ophthalmic Physiol Opt 2016;36:240-65.

36. Latham K, Baranian M, Timmis M, Pardhan S. Emotional Health of People with Visual Impairment Caused by Retinitis Pigmentosa. PLoS One 2015;10:e0145866. 
Figure Legends:

Figure 1: Clinical diagnoses of the study population $(n=50)$.

Figure 2: Raw data for right eyes $(A)$ and left eyes $(B)$. The triangles are datapoints where one or both tests could not provide a quantitative result. The crosses represent BRVT tests that use a combination of E optotype and grating stimuli. The circles represent the rest of the dataset.

Figure 3: Comparison of time taken for both eyes using the Freiburg Acuity Test (FrACT) testing (median time 9 minutes, IQR 6.5 - 11.8 minutes, range 2.8 - 30.0 minutes and Berkeley Rudimentary Vision Test (BRVT) (median time 7 minutes, IQR 4.7 - 9.2 minutes, range $1.0-18$ minutes). BRVT was significantly faster (Wilcoxon signed rank test $P=$ $0.002)$.

Figure 4: Bland-Altman plots examining correlation between Berkeley Rudimentary Vision Test (BRVT) and the Freiburg Acuity Test (FrACT) visual acuity (VA) to calculate coefficient of repeatability $(\mathrm{CR})$. The back dots represent eyes with quantitative measurements and were used in the calculations. The grey dots represent eyes that could not be quantified on one or both tests. The grey long dashed line and corresponding value represents the mean difference between the tests. The short dashed black lines and corresponding values represent the limits of agreement. The grey dotted lines represent the clinically acceptable difference of 0.2 logMAR as discussed in the manuscript. (A) BRVT and FrACT VA difference between right eyes (mean difference $=+0.21, \mathrm{CR}=0.75)$. (B) BRVT and FrACT VA difference between left eyes (mean difference $=+0.17, \mathrm{CR}=0.84$ ). 
Figure 5: (A) Bland-Altman plot comparing better monocular visual acuity (VA) to binocular VA using the Freiburg Acuity Test $($ FrACT) (mean difference $=-0.01, C R=0.79)$. $(B)$ Bubble plot demonstrating spread of VA in better and worse eyes compared to binocular VA. There is no visible difference between the groups in peripheral loss. In patients with central loss, there is a trend with improved binocular VA and reduced spread but this was not significant and individual eyes went in both directions.

Figure 6: (A) Breakdown of visual tasks questionnaire composition into types of daily task (navigation, household, administration and social) and into gross (black portion of bar) and fine (light grey portion of bar) tasks. (B) Bar chart showing percentage of positive responses to the questionnaire (y axis) plotted against VA both eyes FrACT and VA better eye BRVT under bright and dim light conditions. The population is divided into two subgroups: those with VA better than 2.0 as measured on the FrACT (37 eyes) or BRVT (33 eyes), and those with VA worse than or equal to 2.0 as measured on the FrACT (13 eyes) or BRVT (17 eyes). Error bars represent standard error.

Figure 7: Questionnaire responses (A) Person plot demonstrating distribution of population visual acuities (dark bars extending to left) with corresponding summed questionnaire responses in each visual acuity bracket (light grey shaded box extending to right). $\mathrm{N}=40$. The higher the visual acuity logMAR score (i.e. the worse the visual acuity), the more positive responses on the questionnaire. (B) Waterfall chart showing individual responses to questionnaires. Each bar represents an individual's positive responses (expressed as a percentage) to questionnaire tasks relating to navigation (red boxes), household (green boxes), administrative (yellow boxes), and social tasks (blue boxes). The clustering of the blue bars to the left indicates that participants tended to be able to complete a disproportionately higher $\%$ of social tasks than of the other task categories. 

Table 1. Characteristics of the study population. The combined population is shown as well as the subpopulations determined by type of vision loss.

\begin{tabular}{|c|c|c|c|}
\hline & Whole cohort & $\begin{array}{l}\text { Primary peripheral } \\
\text { visual loss }\end{array}$ & $\begin{array}{l}\text { Primary central } \\
\text { visual loss group }\end{array}$ \\
\hline Number & 50 & 24 & 19 \\
\hline Mean age & $63.5(31-95)$ & $54(31-77)$ & $78(55-92)$ \\
\hline Male/female & $20 / 30$ & $14 / 10$ & $5 / 14$ \\
\hline $\begin{array}{l}\text { Median visual acuity } \\
\text { (FrACT) }\end{array}$ & 1.45 & $1.6(1.40-1.94)$ & $1.21(1.01-1.75)$ \\
\hline $\begin{array}{l}\text { Median visual acuity } \\
\text { (BRVT) }\end{array}$ & 1.4 & $1.6(1.4-2.05)$ & $1.4(1.40-1.80)$ \\
\hline Diagnoses (Figure 2) & $\begin{array}{l}\text { AMD } 17 \\
\text { RP } 19 \\
\text { DR } 4 \\
\text { Glaucoma } 3 \\
\text { Miscellaneous } 7 \\
\text { (includes } 7 \text { patients } \\
\text { not classified as } \\
\text { either primary } \\
\text { peripheral or central } \\
\text { loss) }\end{array}$ & $\begin{array}{l}\text { RP } 18 \\
\text { DR } 3 \\
\text { Miscellaneous } 3\end{array}$ & $\begin{array}{l}\text { AMD } 16 \\
\text { DR } 1 \\
\text { Miscellaneous } 2\end{array}$ \\
\hline $\begin{array}{l}\text { Number tested BRVT } \\
\text { first/FrACT first }\end{array}$ & $25 / 25$ & $12 / 12$ & $9 / 10$ \\
\hline $\begin{array}{l}\text { Activities of daily } \\
\text { living questionnaire } \\
\text { score in bright light }\end{array}$ & $27(21.25-33)$ & $32 / 44(24.5-39)$ & $23 / 44(18.5-27)$ \\
\hline $\begin{array}{l}\text { Activities of daily } \\
\text { living questionnaire } \\
\text { score in dim light }\end{array}$ & $35.5(29-39)$ & $37.5 / 44(32-41)$ & $33 / 44(33-38)$ \\
\hline
\end{tabular}


Table 2: Comparison between number of eyes graded with a numerical VA by Freiburg

Acuity Test (FrACT) compared to the Berkeley Rudimentary Vision Test (BRVT)

testing. FrACT gave a much higher number of numerical VAs than the BRVT.

\begin{tabular}{|l|l|l|l|l|l|}
\hline Visual & Number of & Number with & Number with & Number with & No \\
clinical & eyes & FrACT & BRVT & both FrACT and & numerical \\
notes & & numerical & $\begin{array}{l}\text { numerical } \\
\text { VA only }\end{array}$ & VA only & VA numerical \\
VA & & 24 & 0 & 45 & VA \\
\hline Full cohort & 70 & 0 & 0 & 23 & 1 \\
\hline CF & 23 & 6 & 0 & 20 & 0 \\
\hline HM & 27 & 18 & 0 & 2 & 0 \\
\hline LP & 20 & & & & 1 \\
\hline
\end{tabular}




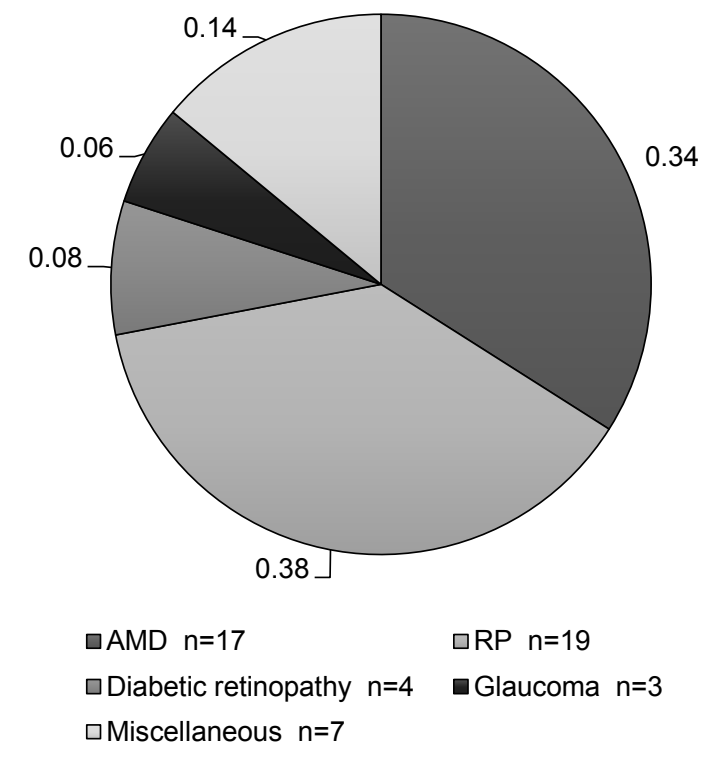

Figure 1

Figure 1 reformattedKAZ

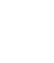

.

$\square$ Diabetic retinopathy $n=4 \quad$ Glaucoma $n=3$
$\square$ Miscellaneous $n=7$ 


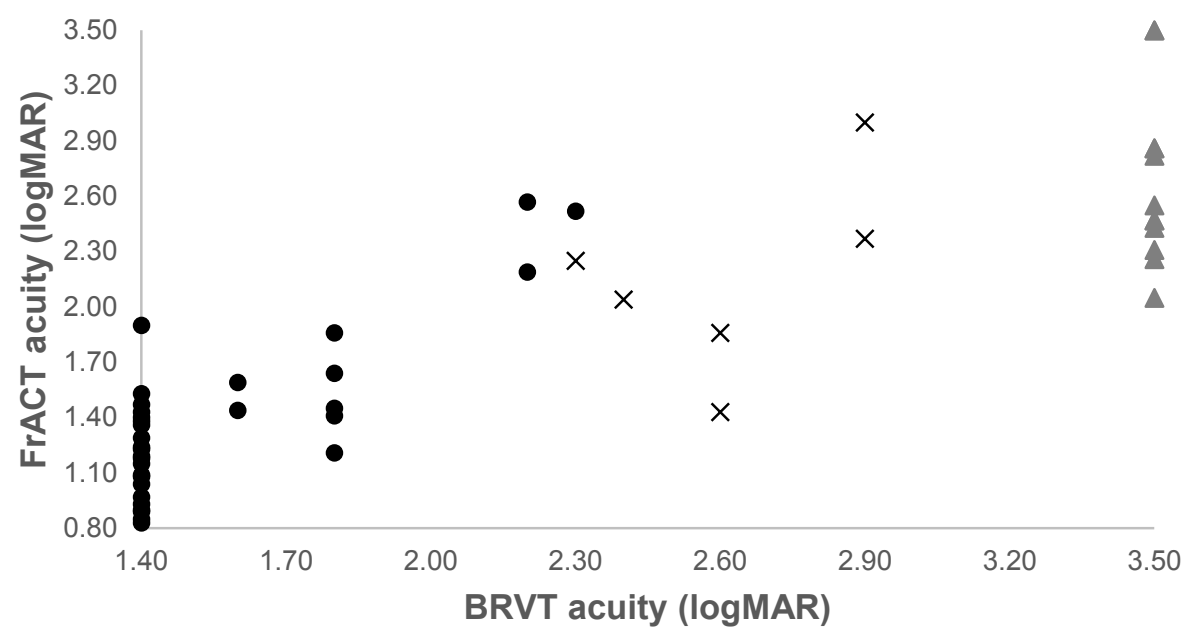

- Main dataset $\quad \times$ BRVT $>2.3 \Delta$ One or both tests not quatifiable

B

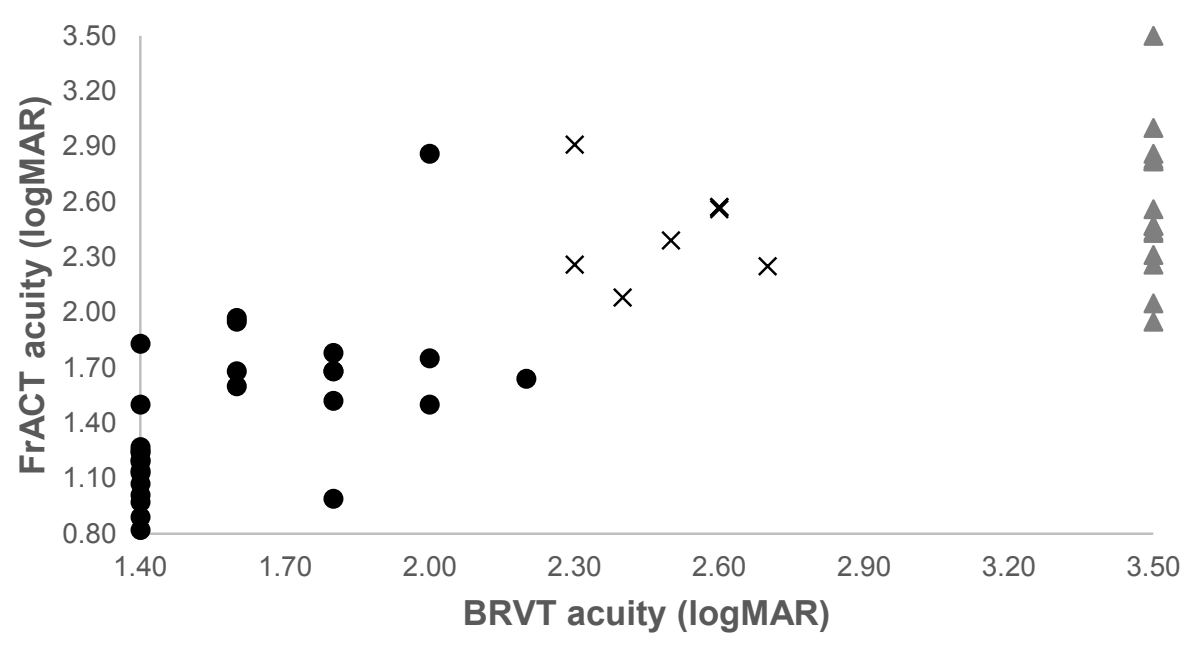

- Main dataset $\quad \times$ BRVT $>2.3 \Delta$ One or both tests not quantifiable 


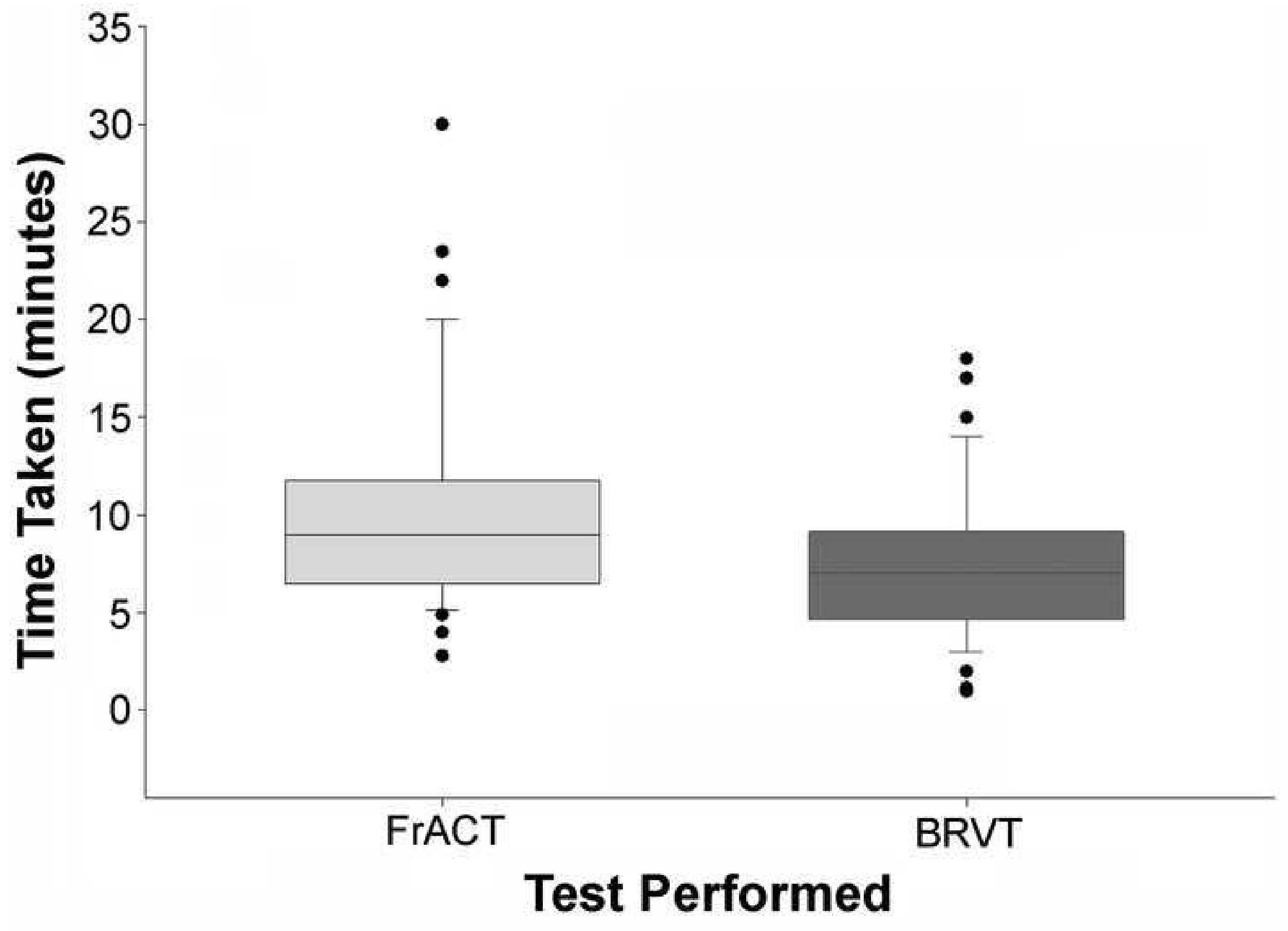




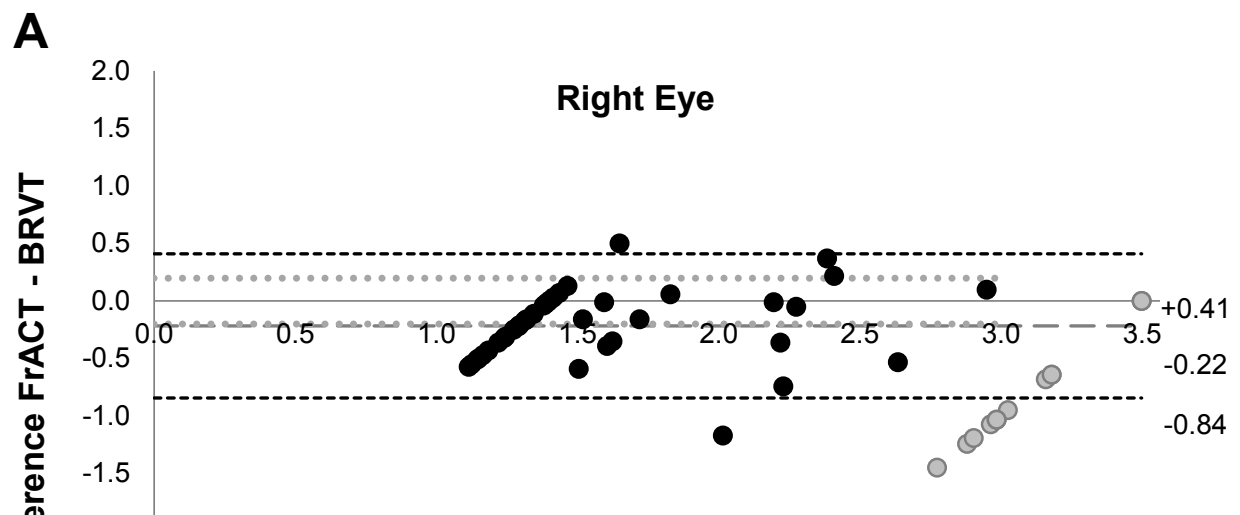

Average of FrACT and BRVT

- Vision measurable in both eyes - Vision not measurable in both eyes - - Mean -.----- Limit of agreement

...... Clinically acceptable change

B

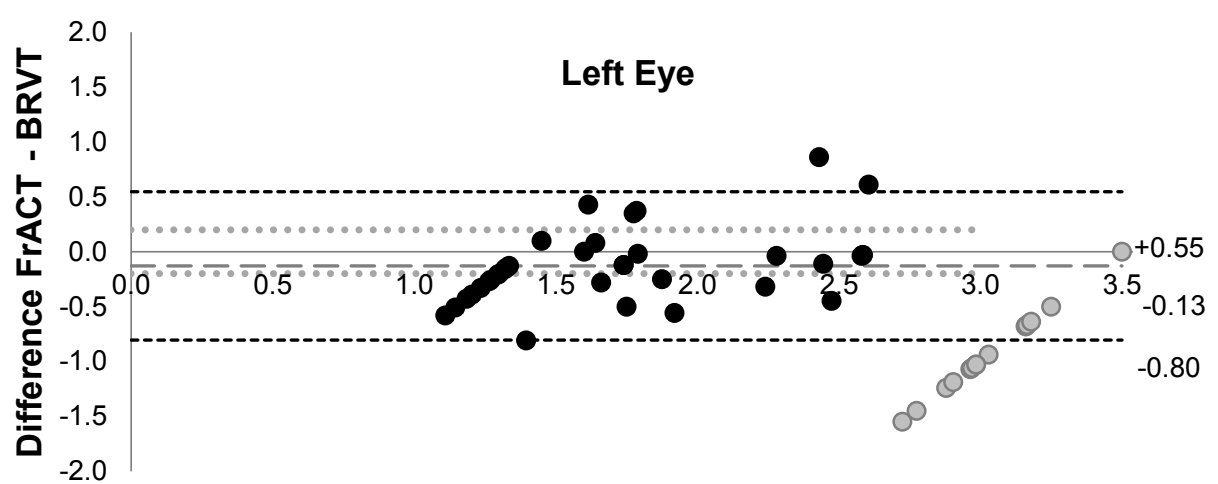

Average of FrACT and BRVT

- Vision measurable in both eyes

- Vision not measurable in both eyes

- - Mean Limit of agreement

...... Clinically acceptable change 

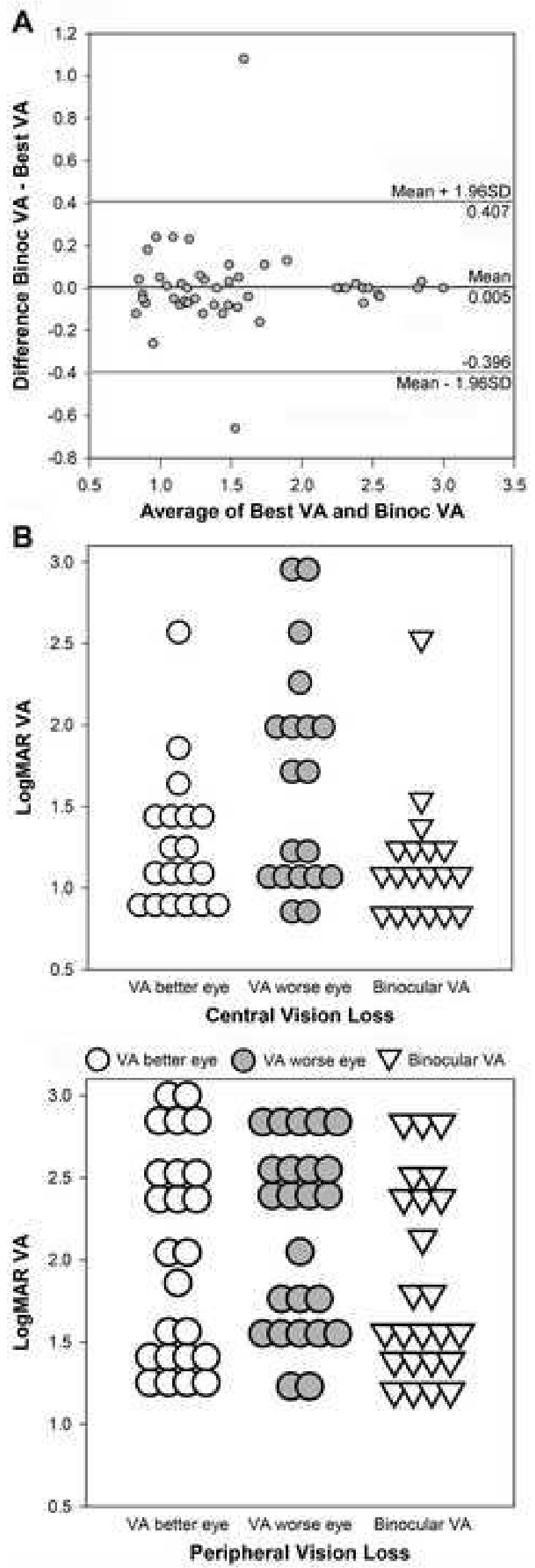
A
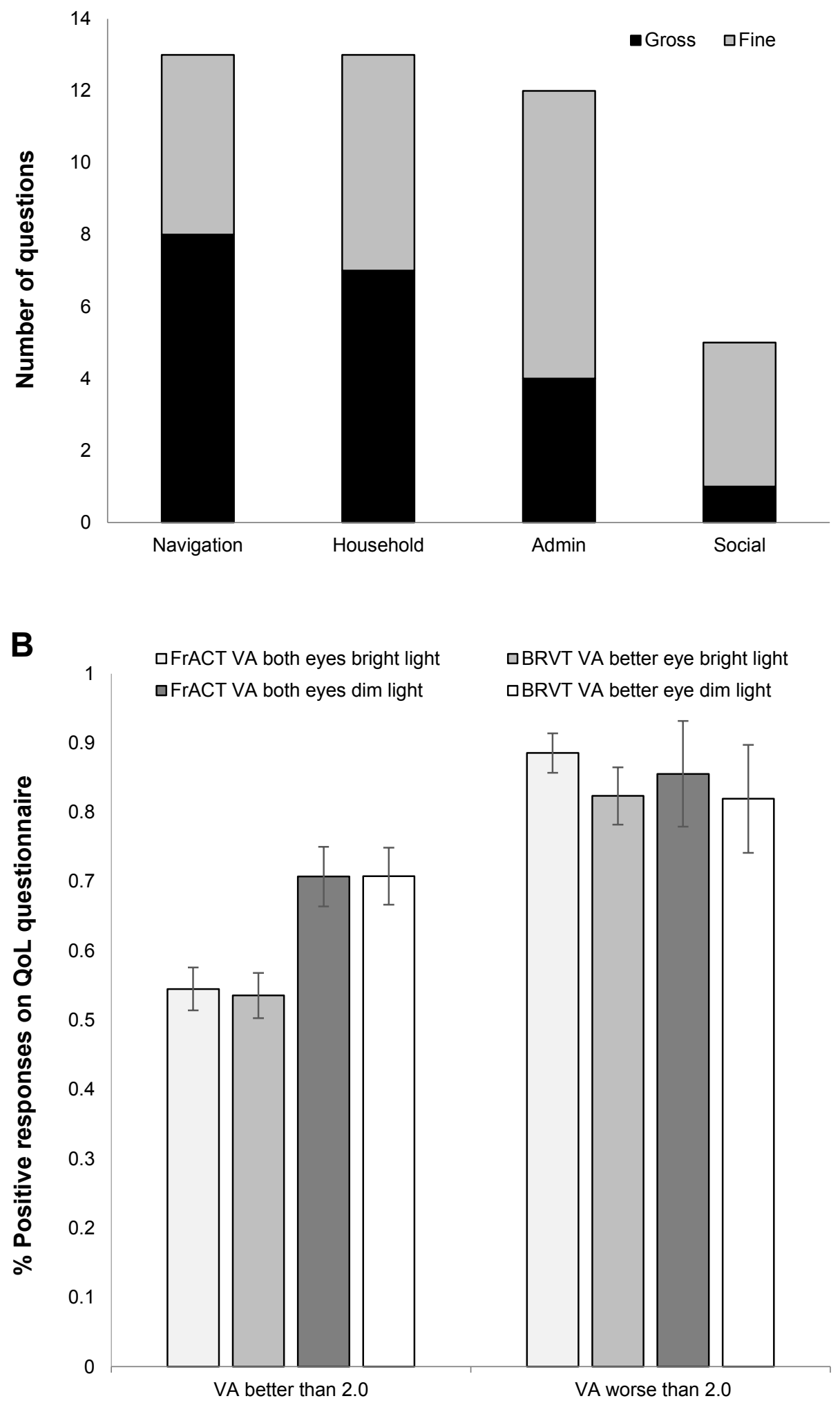

Figure 5 
A

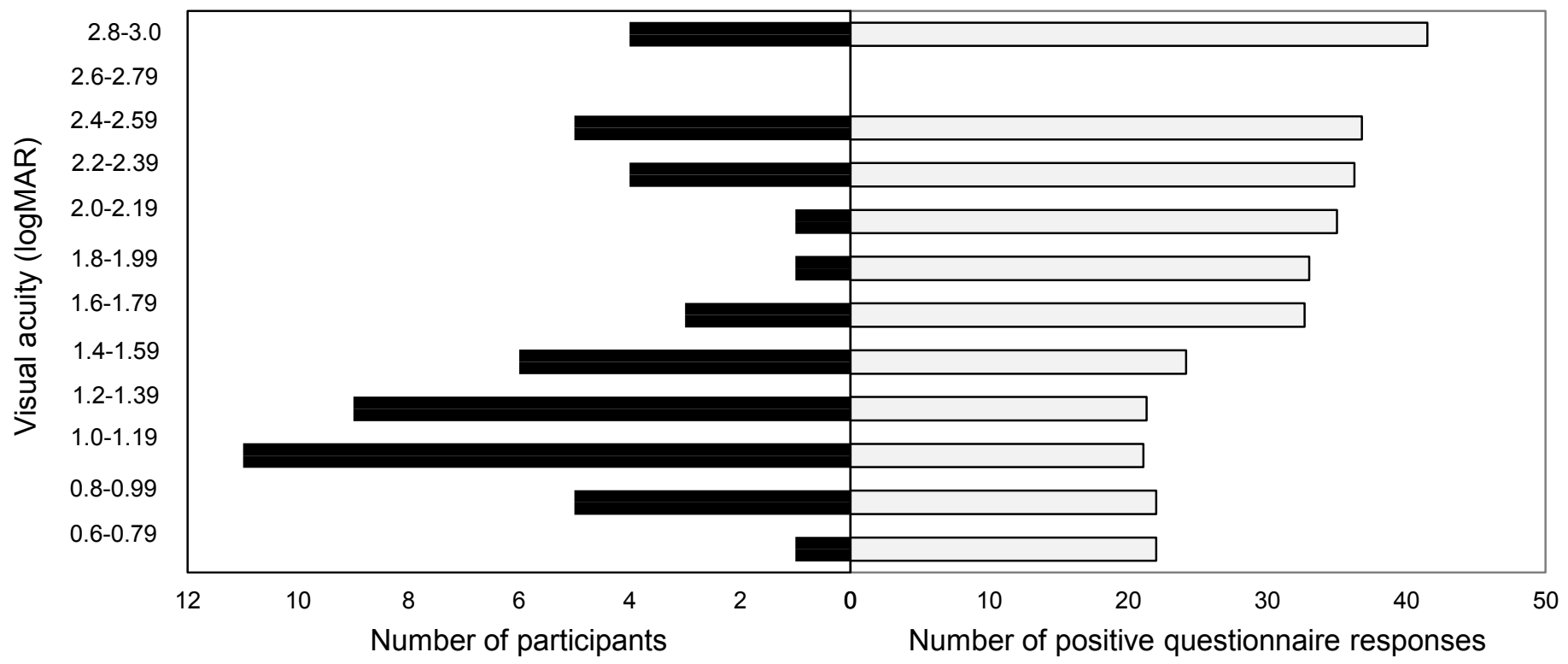

B

:

$\begin{array}{ll} & \\ & 80 \\ & 70 \\ & 60 \\ & 50 \\ & 40 \\ & 30 \\ & 0 \\ & 0\end{array}$
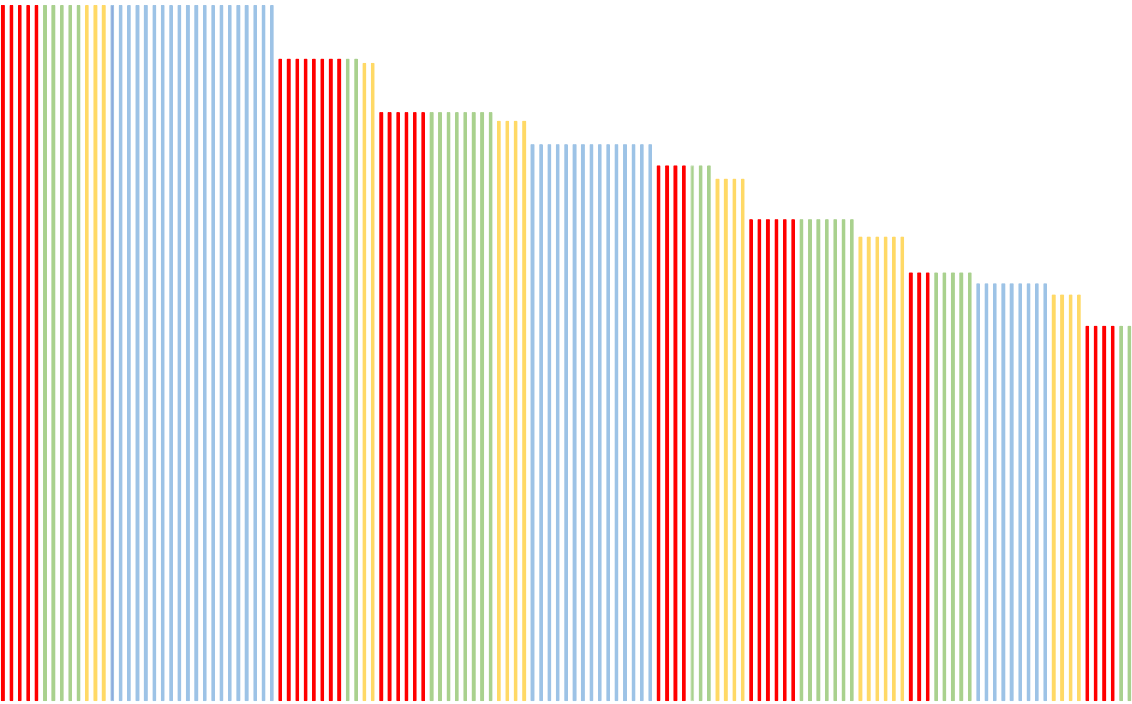

Figure 6 


\section{Daily Living Tasks in Low Vision Survey:}

Does your vision stop you doing any of the following? If task stopped for reasons other than vision or is not relevant, tick NA. If task is still performed by patient, question further to establish whether vision used or task completed through other means such as habit.

\begin{tabular}{|c|c|c|c|c|c|c|}
\hline \multirow[t]{2}{*}{ Task } & \multicolumn{3}{|c|}{ Bright light } & \multicolumn{3}{|c|}{ Dim light } \\
\hline & $\mathbf{Y}$ & $\mathbf{N}$ & NA & $\mathbf{Y}$ & $\mathbf{N}$ & NA \\
\hline $\begin{array}{l}\text { See doorways \& windows } \\
\text { when navigating buildings } \\
\text { indoors }\end{array}$ & & & & & & \\
\hline $\begin{array}{l}\text { Identifying doorways when } \\
\text { outside }\end{array}$ & & & & & & \\
\hline $\begin{array}{l}\text { Identify objects are out of } \\
\text { place }\end{array}$ & & & & & & \\
\hline See faces at arm's length & & & & & & \\
\hline $\begin{array}{l}\text { Walk in a park without } \\
\text { tripping }\end{array}$ & & & & & & \\
\hline $\begin{array}{l}\text { Recognize seasonal } \\
\text { changes in the garden }\end{array}$ & & & & & & \\
\hline Gardening & & & & & & \\
\hline $\begin{array}{l}\text { Attend health appointments } \\
\text { (include full process) }\end{array}$ & & & & & & \\
\hline $\begin{array}{l}\text { Go food shopping in a } \\
\text { familiar supermarket }\end{array}$ & & & & & & \\
\hline $\begin{array}{l}\text { Go shopping for clothes and } \\
\text { other items in unfamiliar } \\
\text { shops }\end{array}$ & & & & & & \\
\hline Internet shopping & & & & & & \\
\hline
\end{tabular}




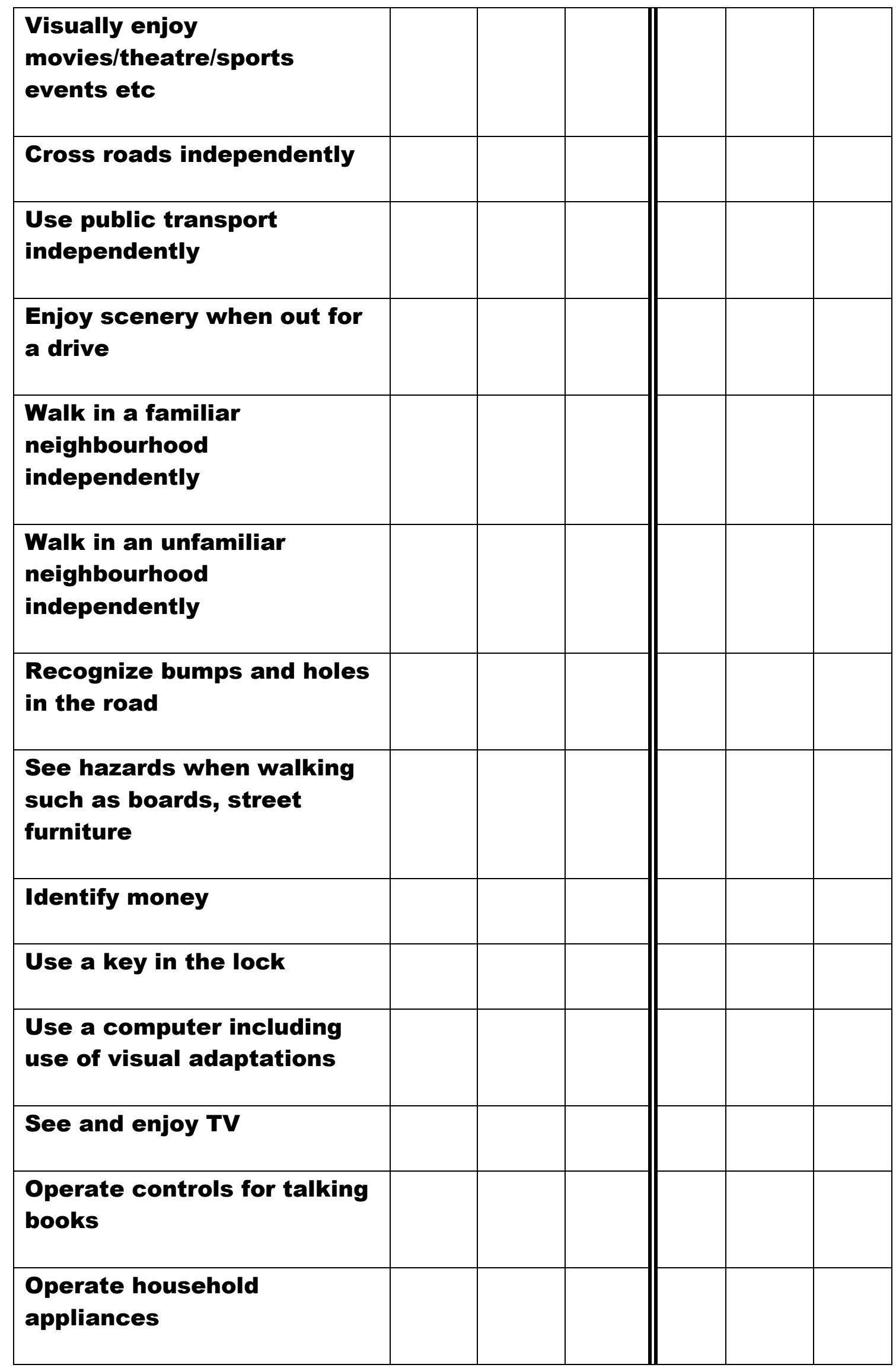




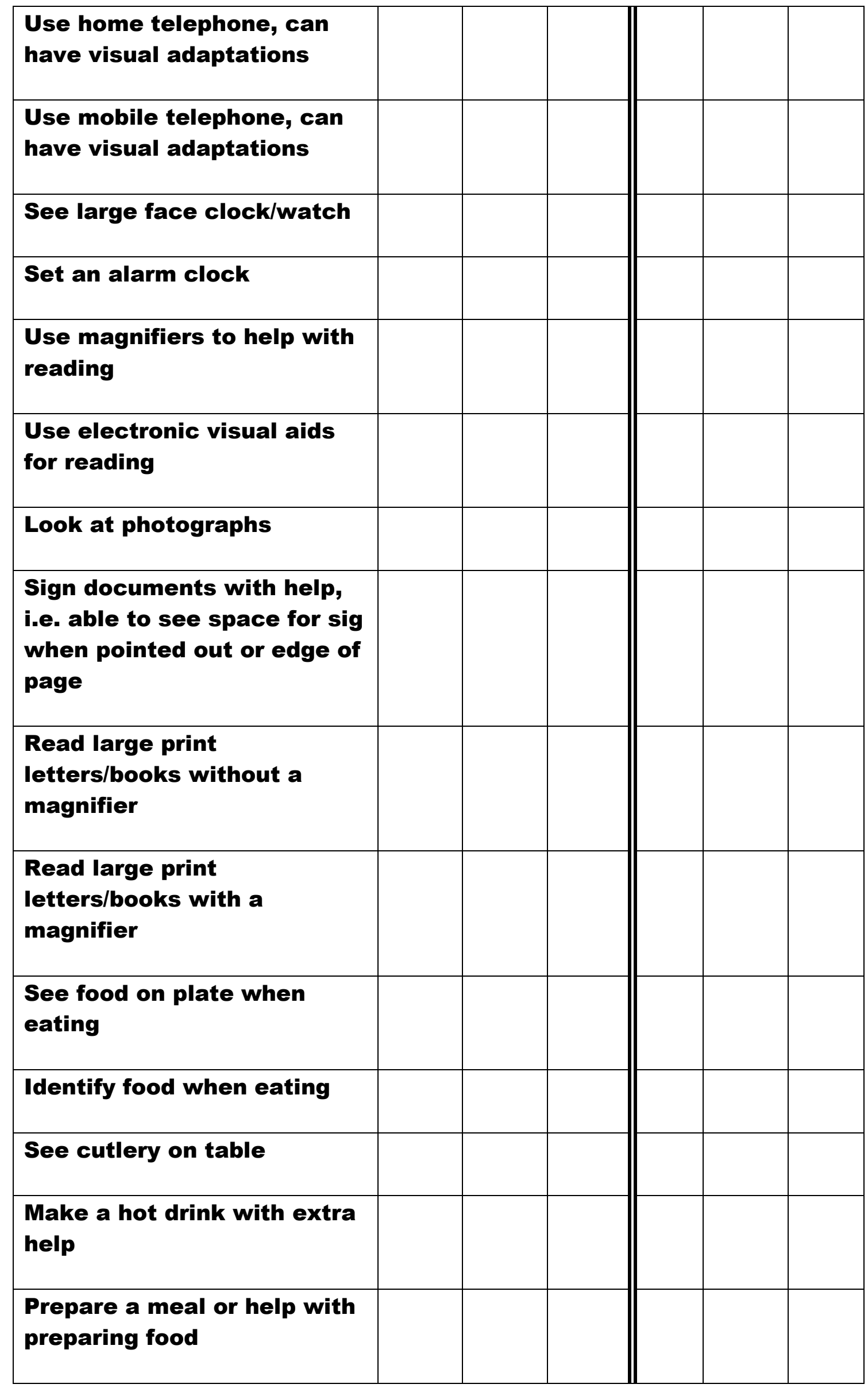




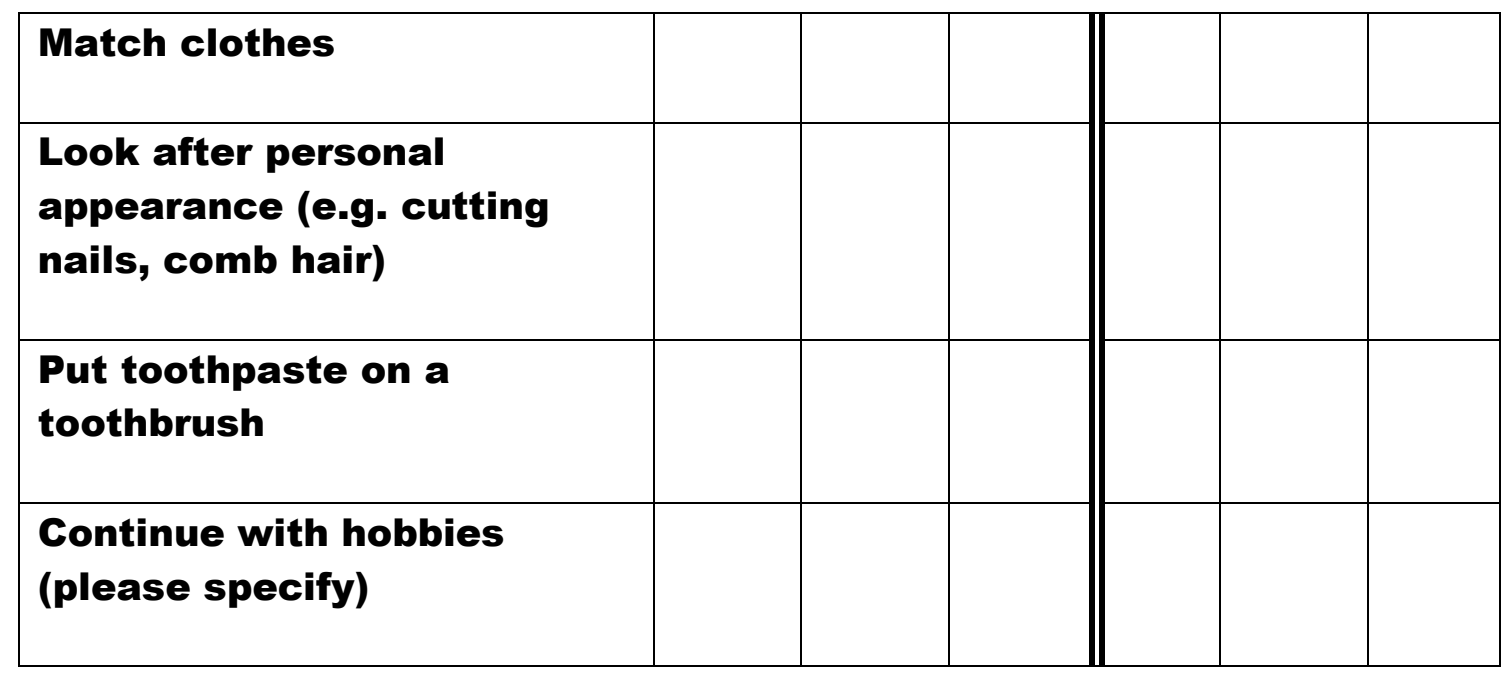

For each task, question patient carefully to establish effect of vision apart from other senses. 


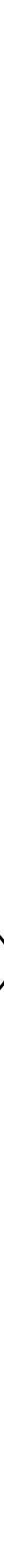




\begin{tabular}{|l|l|l|l|}
\hline Test & Test Distance 100cm & Test Distance 50cm & Test Distance 25cm \\
\hline BRVT & 0.84 & NA & 0.94 \\
\hline FrACT & 0.97 & 0.97 & 0.98 \\
\hline
\end{tabular}

